\title{
Learning Stochastic Finite Automata from Experts
}

\author{
Colin DE LA HIGUERA, \\ EURISE, Université de Saint-Etienne, France \\ www.univ-st-etienne.frleurise/cdlh.html
}

\begin{abstract}
We present in this paper a new learning problem called learning distributions from experts. In the case we study the experts are stochastic deterministic finite automata (sdfa). We deal with the situation arising when wanting to learn sdfa from unrepeated examples. This is intended to model the situation where the data is not generated automatically, but in an order dependent of its probability, as would be the case with the data presented by a human expert. It is then impossible to use frequency measures directly in order to construct the underlying automaton or to adjust its probabilities. In this paper we prove that although a polynomial identification with probability one is not always possible, a wide class of automata can successfully, and for this criterion, be identified. As the framework is new the problem leads to a variety of open problems.
\end{abstract}

Keywords: identification with probability one, grammatical inference, polynomial learning, stochastic deterministic finite automata.

\section{Introduction and Related work}

Inference of deterministic finite automata (dfa) or of regular grammars is a favourite subject of grammatical inference. It is well known [Gol67, Gol78] that the class cannot be identified in the limit from text, i.e. from positive examples only. As the problem of not having negative evidence arises in practice, different options as how to deal with the issue have been proposed. Restricted classes of dfa can be identified [Ang82, GV90], heuristics have been proposed [RV87] and used for practical problems in speech recognition or pattern recognition [L\&al94], and stochastic inference has been proposed as a mean to deal with the problem [CO94, SO94].

Stochastic grammars and automata have been used for some time in the context of speech recognition [RJ93, Ney95]. Algorithms that (heuristically) learn a context-free grammar have been proposed (for a recent survey see [Sak97]), and other algorithms (namely the forward-backward algorithm for Hidden Markov Models, close to stochastic finite automata or the inside-outside algorithm for stochastic context-free grammars) that 
compute probabilities for the rules have been realised [RJ93, LY90]. But in the general framework of grammatical inference it is important to search for algorithms that not only perform well in practice, but that provably converge to the optimal solution, using only a polynomial amount of time.

For the case of stochastic finite automata the problem has been dealt with by different authors: Stolcke and Ohomundro [SO94] learn stochastic deterministic finite automata through Bayes minimisation, Carrasco and Oncina [CO94] through state merging techniques common to classical algorithms for the dfa inference problem. Along the same line Ron et al. [RST95] learn acyclic dfa, proving furthermore that under certain restrictions the inferred automaton is Probably Approximately Correct.

In these papers (but also those from the speech recognition community) an elementary assumption is that the presentation of the examples is unordered (or at least the algorithms do not use this information), and that strings with high probability can appear many times in the learning multisample. If for instance some string has probability $1 / 3$, it is expected that one third of the sample is occupied by this string. This can be justified by practical reasons: when learning from a set where sampling has been done automatically, strings will be repeated according to their probability.

But let us deal with the case where it is not so, and suppose that the protocol does not allow for multiple presentation of data. This is intended to model the situation where the learning data is given to us by a human expert who will certainly not give us repeatedly the same string. Nevertheless the same expert will give us the strings accordingly to the distribution they follow. We can thus consider the expert as a black box containing the distribution to be learned. When requested the expert computes (accordingly to the distribution) a new example and adds it with a label to the learning set, and increases the label.

The expert acts as follows:

rank $\leftarrow 0$

Do $n$ times (or less if less than $n$ strings have non null probability)

1. Generate an unseen example $u$

2. Add ( $u$,rank) to the learning set

3. $r a n k \leftarrow r a n k+1$

The problem of learning from examples delivered by such a black box will be called learning from an expert (as opposed to multisample learning) in the sequel.

In this paper the expert will be a sdfa. We will prove that for restricted classes of sdfa the structure of the automaton can be learned, and the probabilities estimated. Furthermore the algorithms to do so are polynomial in the overall length of the data and convergence can be obtained with probability one. 
In section 2 we provide the elementary definitions and techniques. The problem of learning sdfa involves two different matters: learning the structure or topology of the underlying automaton and estimating the probabilities. In the usual framework of multisample learning both problems are dealt with simultaneously. Two passes are needed in learning from an expert. In section 3 we study the easier of the two problems: estimating the probabilities given a structure. In section 4 we give our results concerning the inference of the structure. We conclude with a first list of open problems concerning this new model.

\section{Preliminaries}

An alphabet is a finite non empty set of distinct symbols. For a given alphabet $X$, the set of all finite strings of symbols from $X$ is denoted by $X^{*}$. The empty string is denoted by $\lambda$. A language $L$ over $X$ is a subset of $X^{*}$. Given $L, \bar{L}$ is the complementary of $L$ in $X^{*}$.

A stochastic deterministic finite automaton ( $s d f a) A=\left\langle X, Q, q_{0}, P, \delta\right\rangle$ consists of an alphabet $X$, set of terminal symbols, a set $Q$ of states, with $q_{0}$ the initial state, a partial transition function $\delta: \mathrm{Q} \times X \rightarrow Q$ and $P$ a probability function $Q \times X \cup\{\lambda\} \rightarrow \mathbb{Q}$, such that:

$$
\forall q \in Q, \sum_{x \in X \cup\{\lambda\}} P(q, x)=1
$$

We define recursively:

$$
\delta\left(q_{i}, x . w\right)=\delta\left(q_{\delta\left(q_{i}, x\right)}, w\right)
$$

And the probability for a string to be generated by $A$ is defined recursively by:

$$
P\left(q_{i}, x . w\right)=P\left(q_{i}, x\right) . P\left(q_{\delta\left(q_{i}, x\right)}, w\right)
$$

The language generated by the automaton is defined as $L=\left\{w \in X^{*}: p(w) \neq 0\right\}$

In case the sdfa contains no useless nodes it generates a distribution over $X^{*}$. We can then also define recursively:

$$
\begin{aligned}
& P\left(q_{i}, x . w X^{*}\right)=P\left(q_{i}, x\right) \cdot P\left(q_{\delta\left(q_{i}, x\right)}, w X^{*}\right) \\
& P\left(q_{i}, X^{*}\right)=1
\end{aligned}
$$

The class of stochastic regular languages consists of all languages generated by stochastic non deterministic finite automata. Although not all stochastic regular languages can be generated by sdfa, we will concentrate on the deterministic case: indeed determinism plays a central part in grammatical inference, and by doing so we are following the same line as related work [CO94, SO94, RST95]

Two sdfa are equivalent if they provide identical probability distribution over $X^{*}$, i.e. if every string over $X$ has equal probability for both distributions.

A sample is a finite presentation of examples, i.e. a subset $S$ of $X^{*}$, and a rank function $\rho: S$-> $\mathbb{N}$ giving the rank of each element of $S$. 
The main tool to compare distributions given a sample is to use the fact that given strings appear before others in the sample. This will be done through the following lemma:

\section{Lemma 1}

Let $A=<X, Q, q_{0}, P, \delta>$ be a sdfa, $S$ a sample and $w, w^{\prime}$ two strings appearing in $S$ such that $w=u v, w^{\prime}=u^{\prime} v$ and $\delta\left(q_{0}, u\right)=\delta\left(q_{0}, u^{\prime}\right)$.

Then $\operatorname{Pr}\left(\rho(w)<\rho\left(w^{\prime}\right)\right)=\frac{P\left(q_{0}, u X^{*}\right)}{P\left(q_{0}, u X^{*}\right)+P\left(q_{0}, u^{\prime} X^{*}\right)}$

\section{Proof}

$$
\begin{aligned}
& \operatorname{Pr}\left(\rho(w)<\rho\left(w^{\prime}\right)\right)=\frac{P\left(q_{0}, w\right)}{P\left(q_{0}, w\right)+P\left(q_{0}, w^{\prime}\right)}= \\
& \frac{P\left(q_{0}, u X^{*}\right) \cdot P\left(q_{\delta\left(q_{0}, u\right)}, v\right)}{P\left(q_{0}, u X^{*}\right) \cdot P\left(q_{\delta\left(q_{0}, u\right)}, v\right)+P\left(q_{0}, u^{\prime} X^{*}\right) \cdot P\left(q_{\delta\left(q_{0}, u^{\prime}\right)}, v\right)}= \\
& \frac{P\left(q_{0}, u X^{*}\right)}{P\left(q_{0}, u X^{*}\right)+P\left(q_{0}, u^{\prime} X^{*}\right)}
\end{aligned}
$$

\section{Learning the probabilities}

In this section we suppose that the actual structure (the automaton) is given, and the problem is to infer from the data the probabilities of the automaton. Thus we are given an automaton $A=<X, Q, q_{0}, P, \delta>$, and a sample $S$.

In the case of repeated data the results for the problem are two fold. On one hand the backward-forward algorithm performs well in practice [LY90], or alternatively Alergia [CO94] can compute the probabilities. On the other hand negative results [AW92, K\&al94] show that if the size of the alphabet is allowed to increase no polynomial algorithm can achieve identification.

We start this section with a counter-example showing that in general the probabilities are hard to learn:

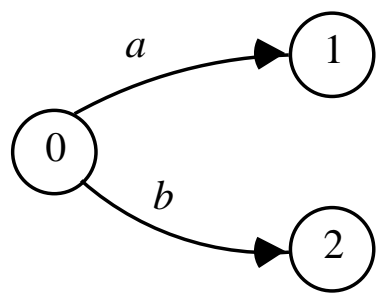

figure 1

When learning from an expert from the automaton of figure 1 a set of learning examples can contain at most 3 examples, and the 6 possible orderings or rank functions 
for these 3 examples will only allow us to learn 6 different distributions. Therefore a necessary condition for learning to be possible is that an infinite number of examples is available. This condition has to hold for all probabilities we wish to compute hence it is sufficient (but also necessary) that the automata has to accept an infinity of strings reentering at least once into the initial state. Technically:

\section{Definition 1}

$$
\begin{aligned}
& \text { A sdfa is left infinite if } \\
& \left|\left\{u \in X^{*}: \delta\left(q_{0}, u\right)=q_{0} \wedge \exists v \in X^{*} P(u v)>0\right\}\right|=\infty
\end{aligned}
$$

In order to learn the probability distributions we need the following notations: given $q \in Q$, a language $L$ over $X$ and a string $u \in X^{*}$,

$$
\begin{aligned}
& \mu(u, L)=\min \{\rho(\mathrm{uv}): v \in L\} \\
& c(q, L)=\left|\left\{w \in X^{*}: \delta\left(q_{0}, w\right)=q \wedge \mu(w, L)<\mu(w, \bar{L})\right\}\right|
\end{aligned}
$$

where if $\mu(w, L)$ is undefined then it is incomparable and so is not counted.

Example : given the following (indexed) presentation of examples on alphabet $X=\{a, b\}$ : $[b b, \lambda, b, b b b a b a b, b a, a b a b a a, b b b$, baabb, bbbaaa, bbbabbb, bababaaaaaaaaaaaabb,

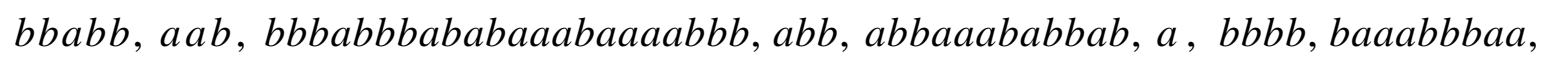
bbaaab, bba, bbaa, abababab]

and the automaton of figure 2 ,

$$
\begin{array}{ll}
\mu(\lambda,\{\lambda\})=1, & \mu(\lambda, \overline{\{\lambda\}})=0 \\
c\left(q_{0},\{\lambda\}\right)=3 & c\left(q_{0}, \overline{\{\lambda\}}\right)=4
\end{array}
$$

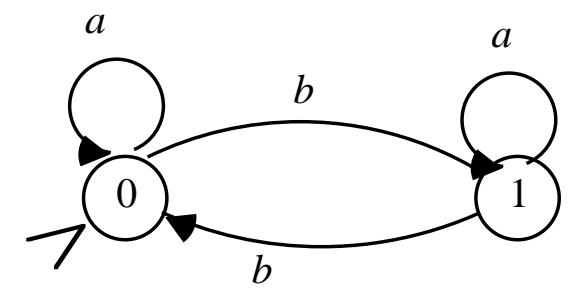

\section{Proposition 1}

figure 2

$$
\begin{aligned}
& 1 \text { when } n \rightarrow \infty, P(q, \lambda)=\frac{c(q,\{\lambda\})}{c(q,\{\lambda\})+c(q, \overline{\{\lambda\}})} \\
& 2 \text { when } n \rightarrow \infty, P(q, a)=\frac{c\left(q, a X^{*}\right)}{c\left(q, a X^{*}\right)+c\left(q, \overline{a X^{*}}\right)} \forall a \in X
\end{aligned}
$$

\section{Proof}

This follows by lemma 1 from the fact that given any string $u$ such that $\delta\left(q_{0}, u\right)=q$ the probability that $\mu(u, L)$ is smaller than $\mu(u, \bar{L})$ is $\frac{P(q, x)}{1-P(q, x)}$ where $x=\lambda$ if $L=\{\lambda\}$, or 
$x=a \in X$ if $L=a X^{*}$. The $\mu(u, L)<\mu(u, \bar{L})$ correspond to the outcome of independent Bernoulli trials, and hence as the number of examples grows, we have the following convergence:

$$
\frac{c(q,\{\lambda\})}{c(q,\{\lambda\})} \text { to } \frac{P(q, \lambda)}{1-P(q, \lambda)} \text { and }(\forall a \in X) \frac{c\left(q, a X^{*}\right)}{c\left(q, \overline{a X^{*}}\right)} \text { to } \frac{P(q, a)}{1-P(q, a)} .
$$

This gives us a formula to estimate the different quantities $P(q, \mathrm{x})$ for each state $q$. The estimation of the probabilities converges if the automaton is left-infinite, i.e. if an unbounded amount of data is available.

Without formalising the computations, all this can be done in polynomial time. From a finite set of examples the probabilities will presumably not add up. It is therefore necessary at the end of the process to adjust the probabilities via :

$$
P^{\prime}(q, x)=\frac{P(q, x)}{\sum_{y \in X \cup\{\lambda\}} P(q, y)}
$$

Following with the example, we have:

$$
\begin{array}{lll}
c\left(q_{1},\{\lambda\}\right)=1 & c\left(q_{1}, \overline{\{\lambda\}}\right)=2 & \\
c\left(q_{0}, a X^{*}\right)=2 & c\left(q_{0}, \overline{a X^{*}}\right)=6 & \\
c\left(q_{0}, b X^{*}\right)=3 & c\left(q_{0}, \overline{b X^{*}}\right)=2 & \\
c\left(q_{1}, a X^{*}\right)=2 & c\left(q_{1}, \overline{a X^{*}}\right)=4 & \\
c\left(q_{1}, b X^{*}\right)=3 & c\left(q_{1}, \overline{b X^{*}}\right)=3 & \\
P\left(q_{0}, \lambda\right)=3 / 7 & P\left(q_{0}, a\right)=1 / 4 & P\left(q_{0}, b\right)=3 / 5 \\
P\left(q_{1}, \lambda\right)=1 / 3 & P\left(q_{1}, a\right)=1 / 3 & P\left(q_{1}, b\right)=1 / 2
\end{array}
$$

After adjusting we get :

$$
\begin{array}{lll}
P\left(q_{0}, \lambda\right)=60 / 179 & P\left(q_{0}, a\right)=35 / 179 & P\left(q_{0}, b\right)=84 / 179 \\
P^{\prime}\left(q_{1}, \lambda\right)=2 / 7 & P^{\prime}\left(q_{1}, a\right)=2 / 7 & P^{\prime}\left(q_{1}, b\right)=3 / 7
\end{array}
$$

giving the following sdfa:

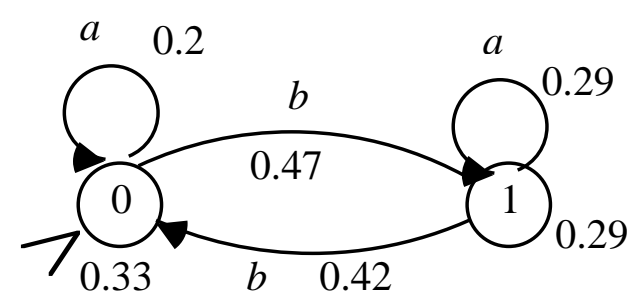

figure 3 


\section{Learning the structure}

In this section we aim at learning the topology of the automaton, given a finite ranked set of examples. In the context of learning from a multisample this is dealt with at the same time as the computation of the probabilities takes place [CO94, RST95, GBE96]. When learning from an expert this cannot be so as and a first pass is needed to construct the underlying automaton. As in the previous section we start with an example showing that for arbitrary automata the topology cannot be inferred. This will lead to defining a restrictive class of sdfa for which we sketch a learning algorithm. The condition we give is sufficient but finding a necessary condition remains an open problem.

\section{Example:}

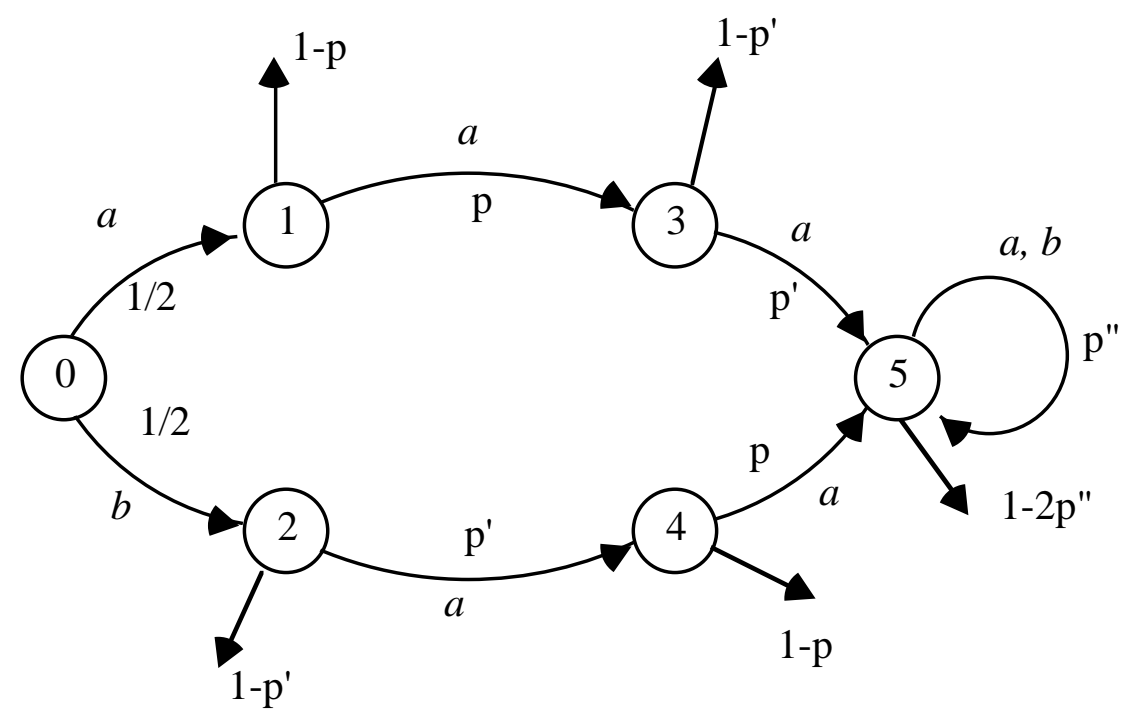

figure 4

Now notice that for all sdfa based on figure 4 such that p.p' is constant the distributions over all strings but $\{a, a a, b, b a\}$ are equal. Thus only a finite number of strings would separate the distribution from the one given by the automaton depicted figure 5 , making identification impossible.

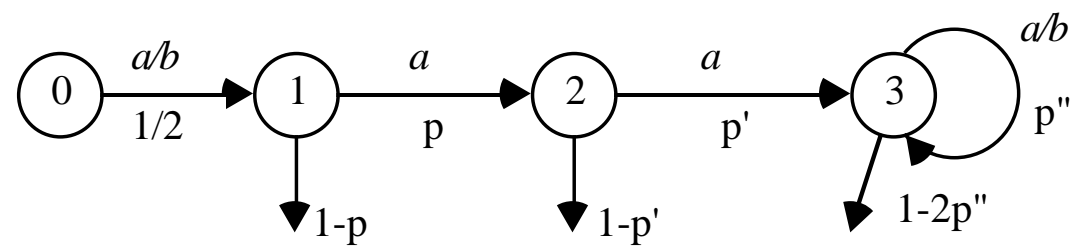

figure 5

The algorithm for learning the structure of a sdfa from an expert is based on Alergia, the algorithm inferring sdfa from multisamples [CO94]. 


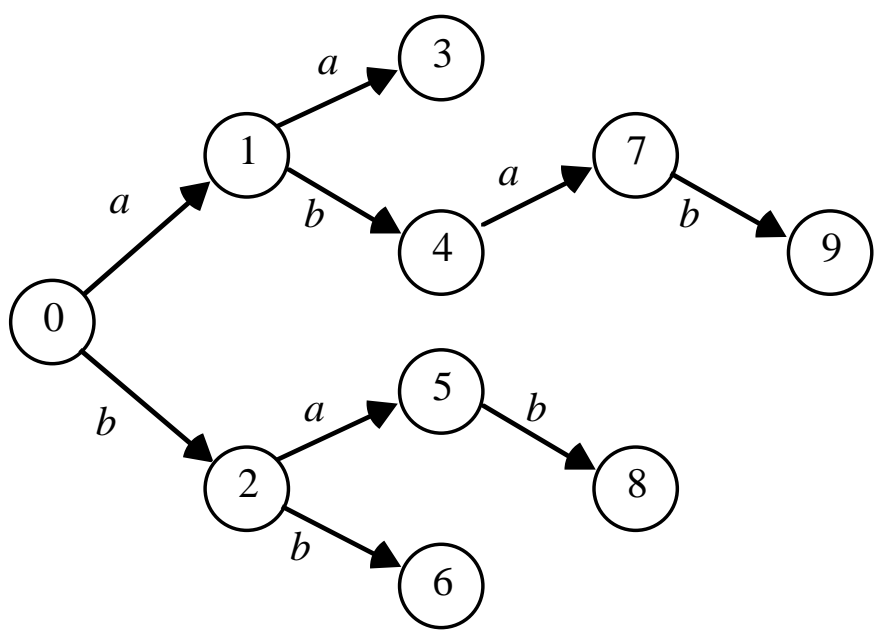

figure 6 .

The prefix tree acceptor for the (ordered) set $[a a, \lambda, a b a b, b, b b, b a b, a b]$. The numbering of the states follows the breadth-first ordering that shall be used in the algorithm.

Initialisation consists in building the prefix tree $T$ from $S$ (the tree that recognises all strings in $S$, an example is depicted figure 6), and the goal is to compute a partition of the states in $T$, such that two states are in the same class if they have to be merged together to obtain the automaton from $T$. To do so a breadth-first exploration of the tree takes place; at each step an attempt is made to merge the new state $q$ with one of the previously visited states $\left(q^{\prime}\right)$. A compatibility function (compatible $\left(q, q^{\prime}\right)$ ) tests how close the distribution of suffixes coming from states $q$ and $q^{\prime}$ are, with respect to a confidence level $\alpha$. In Alergia the test is recursive, as for $q$ and $q^{\prime}$ to be declared compatible, all pairs of states $\langle\delta(q, u)$, $\delta\left(q^{\prime}, u\right)>$, for all $u$ in $X^{*}$, have to be compatible. The number of such tests is only finite, as state $q$ has only a finite number of successors. If the compatibility test returns value true, states $q$ and $q^{\prime}$ are merged.

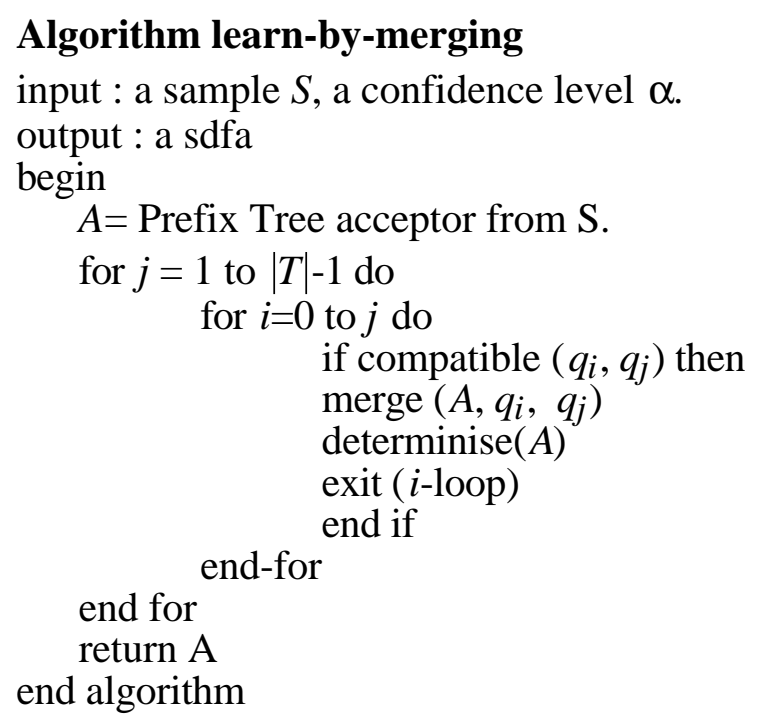


The backbone of the algorithm is identical to that of state merging techniques inferring dfa from positive and negative data [OG94] This enables the algorithm to be modified to take into account quantitative information about the data, thus modifying the exploration order [GBE96, HOV96].

In the case of learning from experts a compatibility test remains to be proposed. To do so it is necessary to translate the order preserving properties into Bernoulli trials. We turn to lemma 1 and define the following:

$$
\begin{aligned}
& \operatorname{minrank}(q, v)=\min \left\{\rho(u v): \delta\left(q_{0}, u\right)=q\right\} \\
& m\left(q, q^{\prime}, L\right)=\left|\left\{v \in L: \operatorname{minrank}(q, v)<\operatorname{minrank}\left(q^{\prime}, v\right)\right\}\right|
\end{aligned}
$$

And using Hoeffding bounds [Hoe63] we have:

$$
\left|P\left(q, q^{\prime}, L\right)-\frac{f}{n}\right|<\sqrt{\frac{1}{2 n} \log \frac{2}{\alpha}} \text { with probability larger than } 1-\alpha
$$

where $P\left(q, q^{\prime}, L\right)$ is the probability that given any string $v$ in $L$, a string $u v$ (with $\delta\left(q_{0}, u\right)=q$ ) appears before the first string $u^{\prime} v$ (with $\left.\delta\left(q_{0}, u^{\prime}\right)=q^{\prime}\right)$ in a sample of $L(A)$;

and where $f=m\left(q, q^{\prime}, L\right), n=m\left(q, q^{\prime}, L\right)+m\left(q^{\prime}, q, L\right)$

As the actual probability is unknown we can use the bound to compare two frequencies We will use a base frequency ( the estimation of $P\left(q, q^{\prime}, X^{*}\right)$ ) to which shall be compared, for each prefix $v$ the frequency corresponding to the value $P\left(q, q^{\prime}, v X^{*}\right)$. This is possible by the following lemma:

\section{Lemma 2}

Let $L$ be a language over $X$. If $q$ and $q^{\prime}$ are in the same class then:

$$
P\left(q, q^{\prime}, X^{*}\right)=P\left(q, q^{\prime}, L\right)
$$

\section{Proof}

$$
\text { We have }\left(\forall v \in X^{*}\right): \frac{\sum: \delta\left(q_{0}, u\right)=q}{\sum P\left(q_{0}, u v\right)}=P\left(q, q^{\prime},\{v\}\right) \text {. The result follows. }
$$

If the difference between frequencies is more than the sum of the confidence ranges the states $q$ and $q^{\prime}$ will be declared incompatible. The actual test is the following:

$$
\left|\frac{m\left(q, q^{\prime}, L_{1}\right)}{n_{1}}-\frac{m\left(q, q^{\prime}, L_{2}\right)}{n_{2}}\right|<\sqrt{\frac{1}{2} \log \frac{2}{\alpha}} \cdot\left(\frac{1}{\sqrt{n_{1}}}+\frac{1}{\sqrt{n_{2}}}\right)
$$

with $n_{i}=m\left(q, q^{\prime}, L_{i}\right)+m\left(q^{\prime}, q, L_{i}\right)$.

We now have the different elements to write our compatibility algorithm. 


\section{Algorithm compatible}

input : $q, q^{\prime}:$ states

output: Boolean

begin

$$
\begin{aligned}
& n_{1} \leftarrow m\left(q, q^{\prime}, X^{*}\right)+m\left(q^{\prime}, q, X^{*}\right) \\
& v a l \leftarrow \frac{m\left(q, q^{\prime}, X^{*}\right)}{n_{1}}
\end{aligned}
$$

do (for all $v \in X^{*}$ such that there exists in $S$ a string $u v w$ with $\delta\left(q_{0}, u\right)=q$ )

$$
\begin{aligned}
& n_{2} \leftarrow m\left(q, q^{\prime}, v X^{*}\right)+m\left(q^{\prime}, q, v X^{*}\right) \\
& \text { if }\left|v a l-\frac{m\left(q, q^{\prime}, v X^{*}\right)}{n_{2}}\right|<\sqrt{\frac{1}{2} \log \frac{2}{\alpha}} \cdot\left(\frac{1}{\sqrt{n_{1}}}+\frac{1}{\sqrt{n_{2}}}\right) \text { return false }
\end{aligned}
$$

end do

return true

end algorithm.

\section{Convergence of the algorithm.}

It is clear that if $q$ and $q^{\prime}$ belong to the same class, compatibility will be detected. But what of the opposite ? Can two states be merged due to a wrong compatibility test ? Clearly, through our initial example the answer is positive. We can nevertheless give a sufficient condition so that two states belonging to two different classes be declared (in probability) incompatible:

\section{Definition 2}

Given a sdfa $A$, two states $q$ and $q^{\prime}$ are order-distinguishable if

$\exists u, v \in X^{*} / P\left(q, q^{\prime}, u X^{*}\right) \neq P\left(q, q^{\prime}, v X^{*}\right)$ and all sets $\left\{w^{\prime} \in X^{*}: \exists w \in X^{*} \delta\left(q_{0}, w\right)=\gamma \wedge\right.$ $\left.P\left(w y w^{\prime}\right) \neq 0\right\}$ (for $\gamma \in\left\{q, q^{\prime}\right\}$ and $y \in\{u, v\}$ ) are infinite.

A sdfa is order-distinguishable if every pair of states in $A$ are order-distinguishable.

It follows that if two states corresponding to two order-distinguishable (classes of) states are tested for compatibility, given sufficient data (which is possible because the amount of possible data is unbounded), the test will return a negative answer. Furthermore the number of calls to function compatible is bounded by $|T| \cdot|T+1| / 2$, where $|T|$ is the size of $T$. And inside each call only $\left|T_{q}\right|$ " $v$ " will be used for tests, where $T_{q}$ is the sub-tree of $T$ at node $q$.

\section{Conclusion}

The results in this paper are two-fold. We first present a new learning paradigm, that we call learning from an expert. Instead of learning a distribution from a multisample we use the order of appearance of the data to do so. We provide the techniques to learn stochastic deterministic finite automata in this context, although to do so we have to restrict 
technically the class. The derived algorithms are polynomial in the size of the learning sample, and they allow for identification with probability one. They do not (it is also the case with multisample learning algorithms [CO94, GBE96]) assure us to identify correctly from a polynomial quantity of data. There are several open questions to be dealt with. First the condition of order-distinguishability is obviously a very strong condition, and if a less restrictive one (or a more descriptive one) for which polynomial identification can be obtained it would be of interest. A second problem is, as in [RTS95] to give sufficient conditions for only a polynomial (in the size of the sdfa) amount of data to be necessary even if this requires restricting further the class of automata. Finally a third trend of research would be to see how having access to more than one expert can help the learning. Perhaps even a second expert can help : one can use the amount of times experts agree on the ordering of specific strings as a compatibility test.

\section{Bibliography}

[AW92] Abe, N. \& Warmuth, M.K. (1992). On the Computational Complexity of Approximating Distributions by Probabilistic Automata. Machine Learning 9, pp. 205 260.

[Ang82] Angluin, D. (1982) Inference of reversible languages. Journal of the ACM 29 (3), pp. 741-765

[CO94] Carrasco, R.C. \& Oncina J. (1994). Learning Stochastic Regular Grammars by means of a State Merging Method. Proceedings of the International Colloquium on Grammatical Inference ICGI-94 (pp. 139-152). Lecture Notes in Artificial Intelligence 862, Springer-Verlag.

[GV90] García, P. \& Vidal, E. (1990). Inference of k-testable languages in the strict sense and application to syntactic pattern recognition. IEEE Transactions on Pattern Analysis and Machine Intelligence 12 (9), pp. 920-925.

[GBE96] Goan, T., Benson, N. \& Etzioni, O. (1996). A Grammar Inference Algorithm for the World Wide Web. In Proceedings of the 1996 AAAI Spring Symposium on Machine Learning in Information Access (MLIA '96), Stanford, CA, AAAI Press.

[Gol67] Gold, E.M. (1967). Language identification in the limit. Inform.\&Control. 10, pp. 447-474.

[Gol78] Gold, E.M. (1978). Complexity of automaton identification from given data. Information and Control 37, pp. 302-320.

[HOV96] de la Higuera, C., Oncina, J. \& Vidal, E. (1996). Identification of dfa : datadependant Vs data-independent algorithms. Proceedings of the International Colloquium on Grammatical Inference ICGI-96 (pp. 313-326). Lecture Notes in Artificial Intelligence 1147, Springer-Verlag.

[Hoe63] Hoeffding, W. (1963). Probability inequalities for sums of bounded random variables. American Statistical Association Journal 58, pp. 13-30.

[K\&al94] Kearns, M., Mansour, Y., Ron, D., Rubinfeld, R., Shapire, R.E. \& Sellie, L. (1994). On the learnability of discrete distributions. In Proceedings of the 24th Annual ACM Symp. on Theory of Computing. 
[LY90] Lari, K. \& Young, S.J. (1990). The estimation of stochastic context free grammars using the inside outside algorithm, Comput. Speech. Language 4, pp 35-56.

[L\&al94] Lucas, S., Vidal, E., Amiri, A., Hanlon, S. \& Amengual, J.C. (1994) A comparison of syntactic and statistical techniques for off-line OCR. Proceedings of the International Colloquium on Grammatical Inference ICGI-94 (pp. 168-179). Lecture Notes in Artificial Intelligence 862, Springer-Verlag.

[N95] Ney, H. (1995). Stochastic grammars and Pattern Recognition. In Speech Recognition and Understanding, edited by P. Laface and R. de Mori, Springer-Verlag, pp. 45-360.

[OG92] Oncina, J. \& García, P. (1992). Inferring regular languages in polynomial time. In Pattern Recognition and Image Analysis, World Scientific.

[RJ93] Rabiner, L. \&Juang, B. H. (1993). Fundamentals of Speech Recognition. PrenticeHall.

[RST95] Ron, D., Singer, Y. \& Tishby, N. (1995). On the Learnability and Usage of Acyclic Probabilistic Finite Automata. Proceedings of COLT 1995, pp 31-40.

[RV87] Rulot, H. \& Vidal, E. (1987). Modelling (Sub)string-Length-Based Constraints through a grammatical Inference Method. In Pattern Recognition : Theory and Applications. Eds: Devijver and Kittler, pp.451-459, Springer Verlag.

[Sak97] Sakakibara, Y. (1997). Recent Advances of grammatical inference. Theoretical Computer Science 185, pp. 15-45.

[SO94] Stolcke, A. \& Omohundro, S. (1994). Inducing Probabilistic Grammars by Bayesian Model Merging. In Proceedings of the International Colloquium on Grammatical Inference ICGI-94 (pp. 106-118). Lecture Notes in Artificial Intelligence 862, Springer-Verlag. 